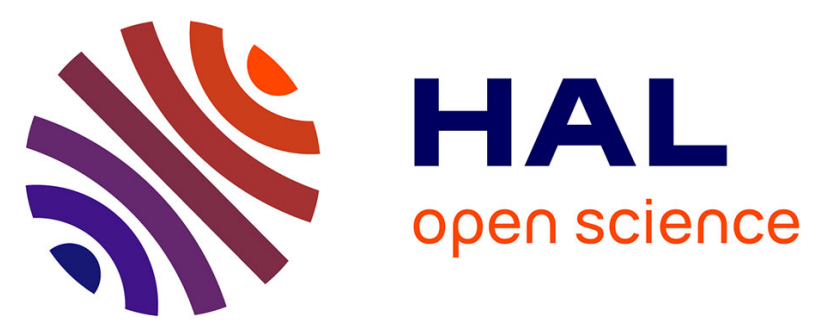

\title{
L'édification de la Basse Plaine de la Salanque (Roussillon, France) au cours de la seconde partie de l'holocène et ses implications sur la répartition des sites archéologiques
}

Jean-Michel Carozza, Carole Puig, Thierry Odiot, Olivier Passarius, Philippe Valette

\section{To cite this version:}

Jean-Michel Carozza, Carole Puig, Thierry Odiot, Olivier Passarius, Philippe Valette. L'édification de la Basse Plaine de la Salanque (Roussillon, France) au cours de la seconde partie de l'holocène et ses implications sur la répartition des sites archéologiques. Quaternaire, 2013, 2 (24), pp.155-165. halshs-00987811

\section{HAL Id: halshs-00987811 \\ https://shs.hal.science/halshs-00987811}

Submitted on 16 Sep 2014

HAL is a multi-disciplinary open access archive for the deposit and dissemination of scientific research documents, whether they are published or not. The documents may come from teaching and research institutions in France or abroad, or from public or private research centers.
L'archive ouverte pluridisciplinaire HAL, est destinée au dépôt et à la diffusion de documents scientifiques de niveau recherche, publiés ou non, émanant des établissements d'enseignement et de recherche français ou étrangers, des laboratoires publics ou privés. 


\title{
L'ÉDIFICATION DE LA BASSE PLAINE DE LA SALANQUE (ROUSSILLON, FRANCE) AU COURS DE LA SECONDE PARTIE DE L'HOLOCÈNE ET SES IMPLICATIONS SUR LA RÉPARTITION DES SITES ARCHÉOLOGIQUES
}

\author{
Jean-Michel CAROZZA ${ }^{1,5}$, Carole PUIG $^{2}$, Thierry ODIOT ${ }^{3}$, Olivier PASSARRIUS ${ }^{4}$ \\ \& Philippe VALETTE 5
}

\begin{abstract}
RÉSUMÉ
Le suivi systématique des travaux d'archéologie préventive ainsi qu'une série de carottages réalisés dans le cadre d'un Programme Collectif de Recherche, ont permis de reconstituer l'évolution de la partie nord de la plaine du Roussillon, la Salanque, entre le Bronze final et la période Moderne.

Quatre lobes principaux sont identifiés: au sud le lobe de Torreilles fonctionne au moins depuis le Bronze final jusqu'à la fin de la période Antique. Il est abandonné au profit du lobe de Saint-Laurent qui est fonctionnel du Ive à la fin du $\mathrm{Ix}^{\mathrm{e}}$ siècle apr. J.C. Les données géoarchéologiques couplées aux sources historiques permettent ensuite de dater la mise en place du lobe de Saint-Hippolyte entre le $x^{e}$ siècle et la seconde moitié du XIII' ${ }^{\mathrm{e}}$ siècle, son abandon pouvant se produire en 1279-1280. Enfin, le lobe actuel dit du Barcarès se développe depuis le XIV e siècle. L'endiguement de l'Agly vers 1334 a contribué à la pérennisation de cette entité unique au cours du Petit Age Glaciaire. La localisation des sites archéologiques est donc fortement dépendante de l'âge des lobes et des dynamiques sédimentaires d'enfouissement associées.
\end{abstract}

Mots-clés : Roussillon, Méditerranée, Agly, delta, architecture alluviale, défluviation, taphonomie, géoarchéologie

\section{ABSTRACT}

ALLUVIAL BUILDING IN THE SALANQUE LOWER PLAINE (ROUSSILLON, FRANCE) DURING THE SECOND PART OF THE HOLOCENE AND ITS IMPLICATIONS ON THE DISTRIBUTION OF ARCHAEOLOGICAL SITES

The systematic monitoring of rescue archaeology excavations coupled with a series of cores carried in the context of a Collective Research Programme founded by Ministery of Culture, helped to reconstruct the evolution of the northern part of the Roussillon plain, the Salanque, between the Middle Bronze Age and the Modern period. Four main lobes are identified: in the south the Torreilles lobe was active since at least the Middle Bronze Age to the end of the Roman period. It was abandoned in favour of SaintLaurent lobe, which is functional between the end of the fourth to the ninth century AD. Geoarchaeological data from these sources and historical data allow us to date the establishment of the Saint-Hippolyte lobe between the tenth century and the second half of the thirteenth century. This lobe was abandoned around 1279-1280 AD. Finally, the Barcarès lobe developed from the fourteenth century. Artificial levee and dike built since 1334 AD on the Agly River contributed to fix this single lobe since the Little Ice Age. The age and location of archaeological sites is thus strongly correlated with lobe ages and with associated alluvial sedimentary processes that led to site burial or destruction.

Keywords: Roussillon, Mediterranean, Agly River, alluvial architecture, avulsion, taphonomy, geoarchaeology

\section{1 - INTRODUCTION}

Les dynamiques de peuplement des basses plaines littorales ont été fortement influencées par les évolutions paléogéographiques qui ont affecté ces espaces au cours de la seconde moitié de l'Holocène. Dans un premier temps l'absence de sites connus a été imputée à des causes sociales («répulsivité» des milieux, type d'organisation des territoires et/ou des réseaux de peuplement, habitat de hauteur; Py, 1993). Ce n'est que récemment

\footnotetext{
${ }^{1}$ Université de Strasbourg, Faculté de Géographie, 3 rue de l'Argonne, F-67000 STRASBOURG. Courriel: carozza@unistra.fr

${ }^{2}$ FRAMESPA - UMR 5136, Maison de la Recherche, 5 allée A. Machado, F-31058 TOULOUSE cedex. Courriel : cpuig@archaeologie-acter.fr

${ }^{3}$ Service Régional de l'Archéologie Languedoc-Roussillon, 5 rue Salle-L'Evêque, F-34000 MONTPELLIER.

Courriel: thierry.odiot@culture.gouv.fr

${ }^{4}$ Service Archéologique Départemental des Pyrénées-Orientales, 74 avenue P. Alduy, F-66000 PERPIGNAN.

Courriel: olivier.passarrius@cg66.fr

${ }^{5}$ GEODE - UMR 5602 CNRS, Maison de la Recherche, 5 allée A. Machado, F-31058 TOULOUSE cedex. Courriel: valette@univ-tlse2.fr
} 
que les biais liés à la paléogéographie (colmatage récent de zones lagunaires ou palustres par exemple ou encore exploitation privilégiée de certains types de milieux) ou aux dynamiques sédimentaires (biais taphonomique de recouvrement) ont été pris en compte afin de corriger l'image du peuplement, principalement acquis par prospection de surface (Berger, 2011). L'intégration de ces données a modifié en profondeur la vision des relations entre les sociétés du passé et l'environnement.

Dans la plaine du Roussillon (fig. 1), la compréhension de l'organisation territoriale des sites et la présence d'espaces vides de toute occupation avant la période Moderne ont été l'un des motifs de la mise en place d'un Programme Collectif de Recherche (PCR) (Carozza, 2005). La faible occupation humaine pré-Moderne de la plaine, notamment à l'aval du méridien de Perpignan, malgré de nombreux diagnostics ou prospections, a fait l'objet de différentes explications. Les hypothèses déterministes liées aux milieux ont été privilégiées par l'archéologie, sans toutefois s'appuyer sur des éléments analytiques précis. Marichal et al. (1997) ont suggéré que l'érosion post-Néolithique pouvait également avoir influencé la préservation des artefacts et la répartition des sites connus. Calvet et al. (2002) ont mis en évidence l'importance de l'alluvionnement récent lié au Petit Âge
Glaciaire et l'effet de masque qu'il pouvait induire. Ces auteurs soulignent toutefois la forte variabilité spatiale de ce recouvrement qui régulariserait une paléotopographie plus différenciée. Celle-ci pourrait être pour partie héritée d'une phase d'incision médiévale entre le $\mathrm{x}^{\mathrm{e}}$ et le milieu du XIII ${ }^{\mathrm{e}}$ siècle (Carozza et al., 2010), qui aurait pu conduire au déstockage d'une partie des sédiments accumulés dans la plaine. Enfin, la préservation différentielle liée au mode d'accumulation et d'empilement des dépôts pourrait également avoir joué un rôle important (Carozza, 2011). Ces différentes hypothèses ne sont pas nécessairement contradictoires et rendent avant tout compte d'échelles d'observation différentes.

Dans ce travail, nous montrons que la répartition des sites archéologiques à l'échelle de la plaine est avant tout contrôlée par l'architecture sédimentaire de la plaine et par le mode d'édification des deltas à haute énergie, plus particulièrement dans le cas de la basse plaine de l'Agly.

\section{2 - MÉTHODES}

Afin de reconstituer l'évolution de la basse plaine de la Salanque, une démarche multi-sources a été mise en œuvre par la combinaison de 3 méthodes:

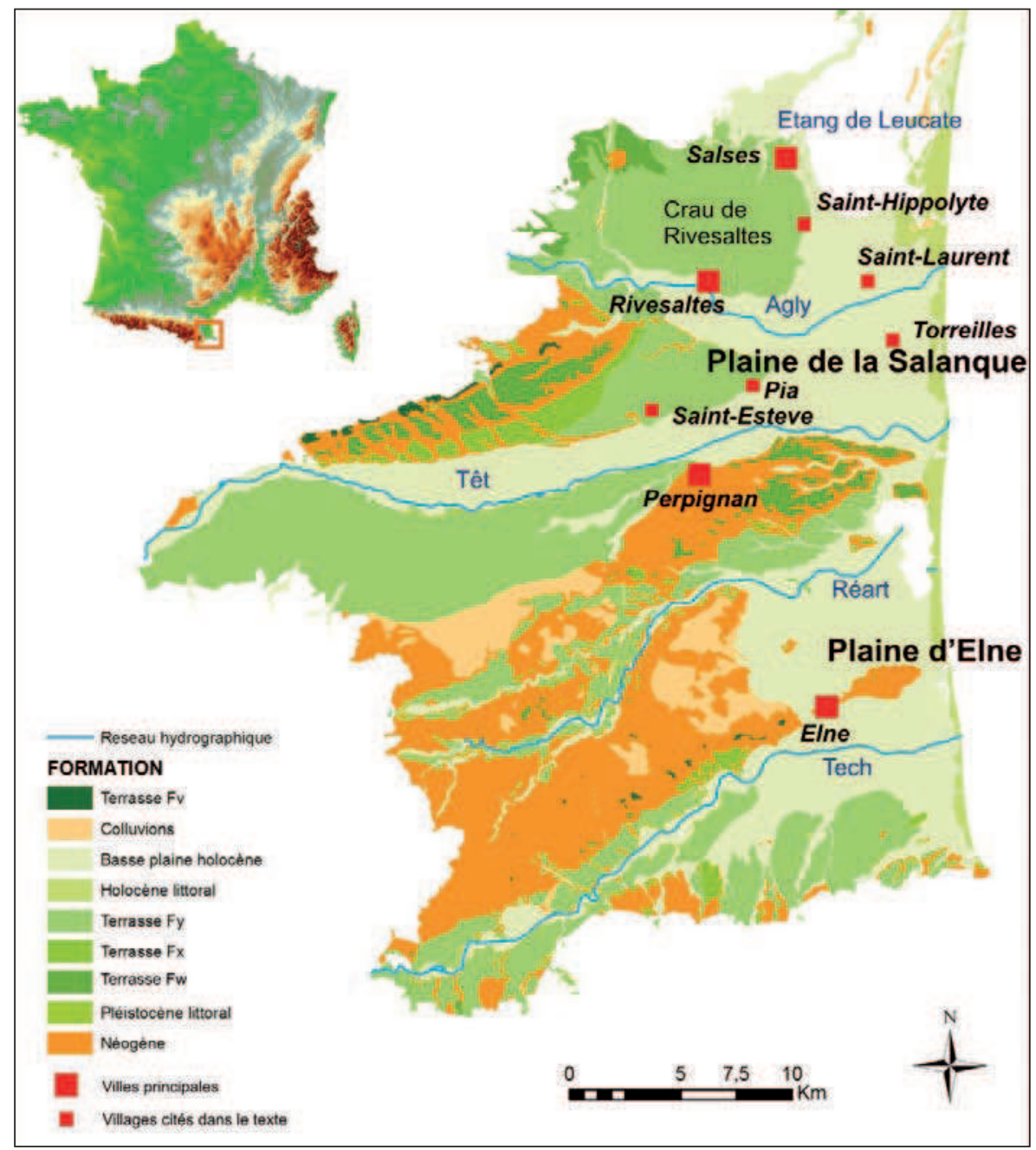

Fig. 1 : Localisation de la zone d'étude.

Fig. 1: Location of the studied area. 
- une approche de la géométrie des dépôts à petite échelle, réalisée par dépouillement systématique de la Banque de Données du Sous-Sol (BDGSS) du BRGM. Les données recueillies sont principalement les faciès sédimentaires décrits sommairement sur les logs de terrain, plus rarement des données de diagraphie. La chrono-stratigraphie de ces formations reste sommaire et distingue généralement le substratum pliocène, les terrains pléistocènes et holocènes. Pour l'Holocène, une distinction entre Holocène marin (sableux ou argileux) et continental est parfois proposée, basée sur la révision de Duvail (2004). Toutefois, certaines attributions restent marquées par une forte incertitude et des confusions entre formations marines, lagunaires et paléosols continentaux épais. Les données chrono-stratigraphiques sont absentes.

- une approche géoarchéologique à partir du suivi d'opérations d'archéologie préventive sur des grands linéaires (RD83 notamment) ou d'interventions ponctuelles (Torreilles) et de carottages mécaniques réalisés à proximité de sites connus. Les carottages ont été effectués à l'aide d'une plateforme APAGEO. Dans les deux cas, l'approche se base sur un relevé systématique de logs lithostratigraphiques et l'identification des principaux faciès pédosédimentaires in situ, incluant les contenus biologiques et archéologiques. Les principales phases d'arrêt ou de ralentissement de la sédimentation enregistrées par des paléosols associés ou non à des occupations ont fait l'objet d'une attention particulière. Un ensemble de datations, issues soit des données archéologiques soit de datations radiocarbones réalisées sur charbons pour le domaine continental ou sur matière végétale pour les formations laguno-marines a également été réalisé afin d'améliorer la résolution chronologique (tab. 1).

- enfin une approche historique, par le dépouillement systématique des données sur la localisation des cours d'eau a été entreprise. Cette approche permet de couvrir les périodes les plus récentes (huit derniers siècles) et notamment d'intégrer l'évolution enregistrée au cours de la transition entre l'Anomalie Climatique Médiévale et le Petit Age Glaciaire (fin $\mathrm{XII}^{\mathrm{e}}$ - début XIV ${ }^{\mathrm{e}}$ siècle). Il s'agit principalement de repérer dans les sources les mentions de «confonts» (limites en vis à vis) entre des propriétés et un cours d'eau qui donne un terminus post quem pour l'abandon des chenaux, ou bien les mentions de chenaux anciens (Agly Vieil ou Vell) qui fournissent alors un terminus ante quem de l'arrêt du fonctionnement. Ici, la résolution chronologique peut atteindre une précision inférieure à la dizaine d'année, parfois même annuelle (Puig \& Carozza, 2012).

L'ensemble de ces informations a ensuite été mis en séquences à différentes échelles spatiales.

\section{3 - ZONE D'ÉTUDE}

La plaine du Roussillon (fig. 1) correspond à une zone de fort élargissement des basses plaines littorales en relation avec le développement d'un bassin sédimentaire néogène de forme trapézoïdale (Calvet, 1994; Carozza, 1998). La plaine est organisée en deux ensembles distincts, séparés par les hautes collines de Perpignan à Canet, qui délimitent la Salanque au nord et la plaine d'Elne au sud. La Salanque est principalement drainée par l'Agly et la Têt, qui constituent les deux hydrosystèmes les plus importants du Roussillon. Du fait d'une alimentation par des bassins versants morphologiquement contrastés, le régime des deux cours d'eau diffère significativement. Ils présentent toutefois comme caractéristique commune de conserver une pente aval forte et un débit à plein bord élevé qui en font des systèmes à haute énergie.

D'un point de vue fonctionnel, la plaine a été divisée en deux entités (Benech \& Mussot, 1995). Dans la partie amont, la plaine fonctionne «à l'érosion » (i.e. les formes alluviales y sont étagées). À l'aval, elle fonctionne «à l'accumulation» (i.e. les formes alluviales aggradent ou progradent). La transition entre les deux domaines est matérialisée par l'intersection entre les profils en long de la terrasse pléistocène la plus récente et des basses plaines holocènes (Carozza, 2011). La terrasse pléistocène passe au-dessous des formations holocènes. Ce point de segmentation correspond généralement à l'apex du système de cônes deltaïques holocènes. De ce fait, les formations holocènes de la Salanque sont limitées au nord par les formations de la Crau de Rivesaltes attribuées au Pléistocène supérieur (Fy1, Clauzon et al., 1989). Au centre de la Salanque, l'interfluve Agly-Têt est bien matérialisé par les terrasses du Pléistocène moyen à supérieur (Fw et Fy) de Saint-Estève et Pia. Vers l'aval, cet interfluve s'abaisse et la limite entre les deux systèmes est alors construite et difficile à identifier d'un point de vue topographique. Par contre, la signature pétrographique des apports de l'Agly par les «sables noirs» remaniés des marnes albiennes, constitue un critère fiable de délimitation de l'influence entre les deux systèmes.

\begin{tabular}{|c|c|c|r|}
\hline Code laboratoire & Nature de l'échantillon & Age conventionnel & Age calibré (2 $\sigma)$ \\
\hline Poz-37118 & Charbon & $340 \pm 30 \mathrm{BP}$ & $1470-1632$ yr AD \\
\hline Poz-37121 & Charbon & $1830 \pm 30 \mathrm{BP}$ & $119-253$ yr AD \\
\hline Poz-37123 & Charbon & $1270 \pm 30 \mathrm{BP}$ & $669-885$ yr AD \\
\hline Poz-37158 & Charbon & $1190 \pm 35 \mathrm{BP}$ & $771-900 \mathrm{yr} \mathrm{AD}$ \\
\hline Poz-37159 & Charbon & $1205 \pm 30 \mathrm{BP}$ & $694-894 \mathrm{yr} \mathrm{AD}$ \\
\hline Poz-37162 & Charbon & $1155 \pm 30 \mathrm{BP}$ & $778-971 \mathrm{yr} \mathrm{AD}$ \\
\hline Poz-27591 & Charbon & $4330 \pm 50 \mathrm{BP}$ & $3011-2857 \mathrm{yr} \mathrm{BC}$ \\
\hline
\end{tabular}

Tab. 1 : Datations ${ }^{14} \mathrm{C}$.

Tab. 1: $C^{14}$ datings 
Le maximum transgressif holocène reste localisé en aval du point de segmentation et sa position dépend principalement de l'importance de l'incision pléniglaciaire. En amont, se développe la plaine alluviale, dominée par les processus fluviaux. Vers l'aval se développent les systèmes progradants et aggradants des basses plaines sensu stricto.

La mobilité des cours d'eau dans la plaine de la Salanque joue un rôle majeur dans l'édification de la plaine et est liée au maintien d'une puissance spécifique forte $\mathrm{y}$ compris à l'aval des systèmes $\left(>40 \mathrm{w} \cdot \mathrm{m}^{-2}\right)$. Elle a fait l'objet de nombreuses hypothèses, notamment en ce qui concerne l'Agly. Sur la base de données de photointerprétation, Guy (in Buscail-Martin, 1978) a suggéré l'existence d'un tracé orienté sud-nord débouchant au niveau de l'anse de la Roquette. Ce tracé aurait été en relation avec l'occupation protohistorique du Port à Salses-le-Château. L'attribution de ce tracé à l'Holocène pose problème (Carozza et al., 2010) et il pourrait correspondre à une position tardiglaciaire en relation avec la migration de l'Agly jusqu'à son axe holocène. Plus récemment Serrat (2000) a proposé un modèle d'évolution de la Salanque en deux phases. L'Agly aurait d'abord édifié un lobe septentrional et se serait jeté dans l'étang de Leucate jusqu'au IV $V^{\mathrm{e}}$ siècle (lobe 1) avant de prendre une direction proche de l'actuel (lobe 2). Ce schéma partiel s'appuie sur peu d'éléments de datation directs.

\section{4 - RÉSULTATS}

\section{1 - AUTOUR DE TORREILLES}

Deux opérations séparées d'environ $500 \mathrm{~m}$ et localisées à l'est de Torreilles ont permis d'appréhender les dynamiques sédimentaires au sud de la zone d'étude (fig. 2).

Sur le site de Gorg d'en Guillem (Corban, 2007), un sondage profond permis de recouper la séquence holocène sur une épaisseur de $6 \mathrm{~m}$ (fig. 3). Elle débute par $2,5 \mathrm{~m}$ d'argile sombre qui n'ont pu être observés que de manière indirecte. Les niveaux inférieurs ont livré des fragments de roseaux qui ont fait l'objet d'une datation radiocarbone. Au-dessus, le niveau compris entre 3,50 et $2,90 \mathrm{~m}$ montre un enrichissement en sables grossiers et renferme un niveau à malacofaune lagunaire. Cette séquence est couronnée par un niveau argileux sombre à structure prismatique qui a livré des fragments de céramique non tournée. Cette unité est localement ravinée

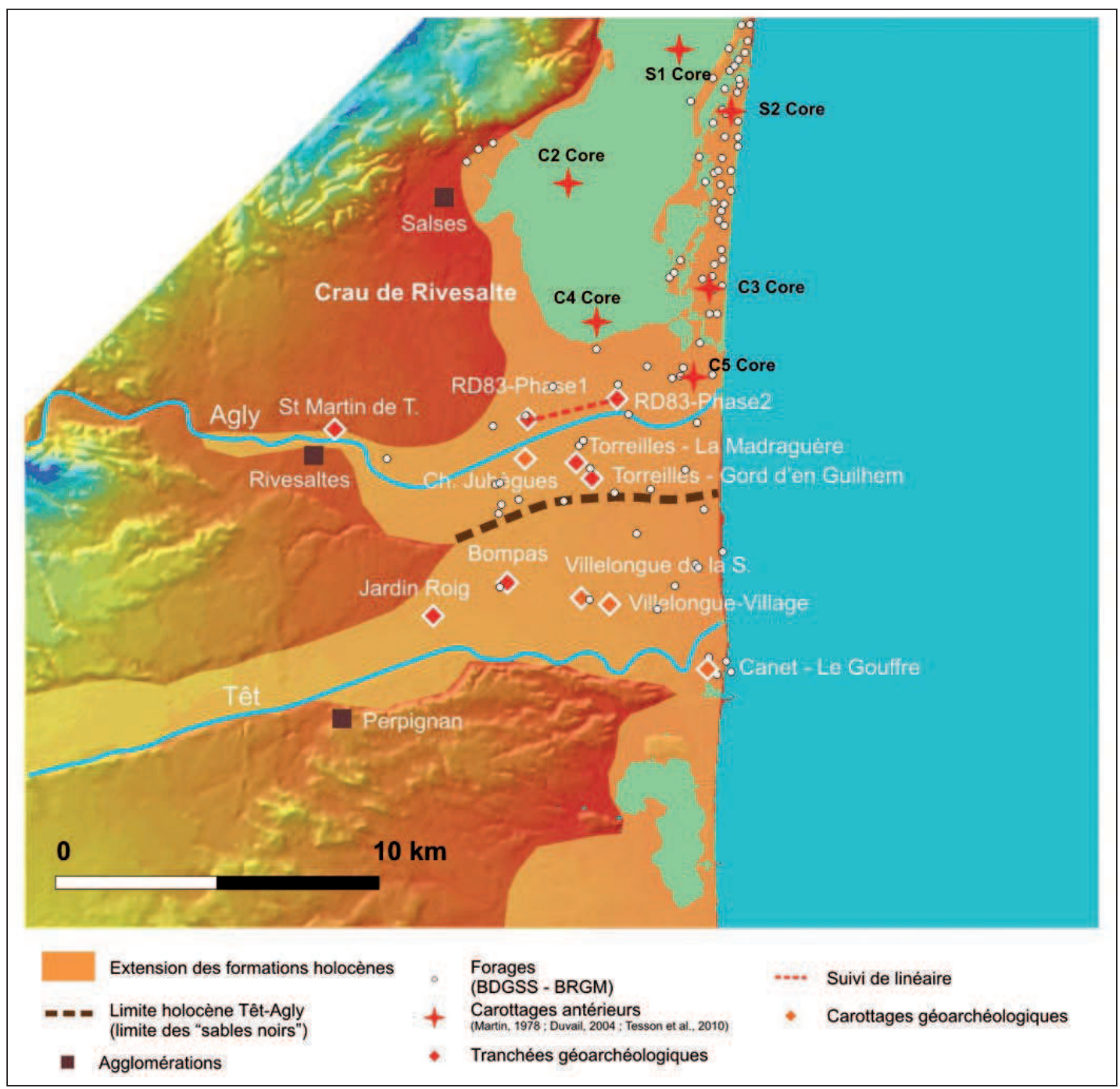

Fig. 2 : Localisation des opérations dans la plaine de la Salanque.

Fig. 2: Location of fieldwork operations in the Salanque plain. 


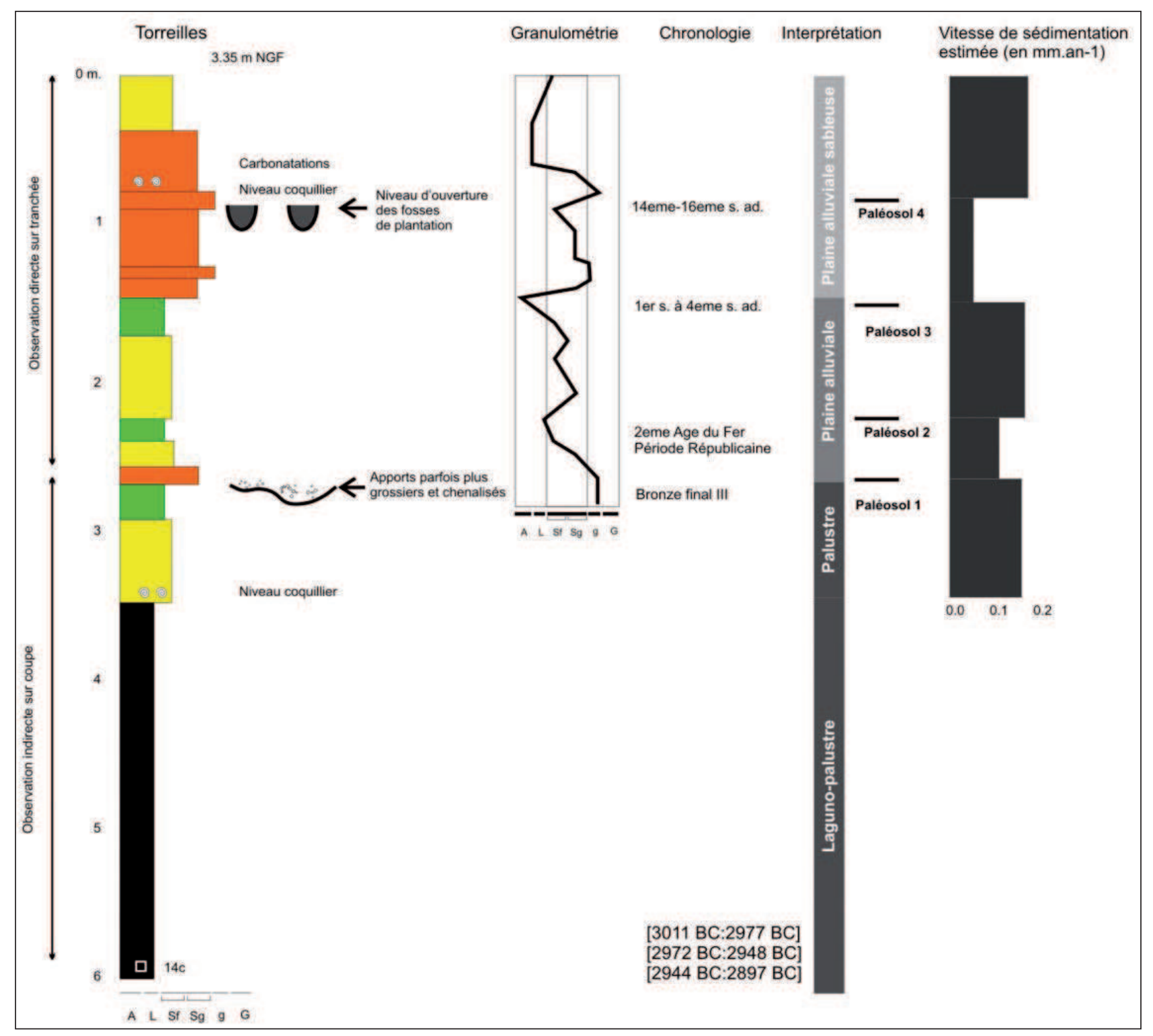

Fig. 3 : Coupe transversale de la tranchée profonde de Gorg d'en Guillem. Fig. 3: Cross-section of the Gorg d'en Guillem trench.

par des apports sableux à sablo-graveleux grossiers, parfois légèrement chenalisés. Cette séquence granodécroissante se termine par un niveau argilo-limoneux sombre qui renferme des fragments de céramique épars. Ce niveau est recouvert par un limon massif de près de $0,60 \mathrm{~m}$ qui a livré à son sommet des artefacts antiques sensu lato. Le sommet de la séquence entre 1,50 m de profondeur et la surface montre un retour à des conditions de dépôt plus dynamiques, dominées par les sables et les sables grossiers. Cette dernière unité renferme un paléosol peu marqué au niveau duquel s'ouvrent des fosses de plantation (paléosol 4 ; fig. 3).

Les travaux menés sur le site de La Madraguère, ont permis une observation plus précise de la séquence supérieure (fig. 4). Ils montrent une bonne corrélation avec les données de Gorg d'en Guilhem. La séquence peut être divisée en 3 unités. À la base, une formation détritique grossière incisée dans des niveaux sableux occupe la partie nord du site. Elle se raccorde latéralement à des niveaux plus fins qui ont livré des éléments de foyers attribués à la Protohistoire (Vial et al., 2009). Le sommet de la séquence n'est préservé que localement. Il a en effet été partiellement érodé par la mise en place de la deuxième unité sédimentaire qui est associée à un paléochenal et une levée de berge à intercalation de niveaux sableux grossiers, fins et limoneux très rythmés, qui témoignent de l'existence d'un chenal confiné. Le milieu de cette séquence renferme un paléosol peu marqué, associé à un niveau d'épandage de matériel antique et qui peut être corrélé au paléosol 3 de la séquence du Gorg d'en Guillem (fig. 3). La séquence supérieure correspond à un ensemble sablo-limoneux, parfois faiblement chenalisé, qui vient sceller un niveau d'ouverture fosses de plantation médiévales.

Ces deux sites permettent de reconstituer l'évolution du secteur sud de la Salanque. Cette zone était occupée entre env. 3000 av. J.-C. et jusque vers env. 1000 av. J.-C par une lagune. L'émersion se produit peu avant le Bronze final III, soit vers 900-850 av. J.-C., en liaison avec une augmentation des apports détritiques. L'appari- 


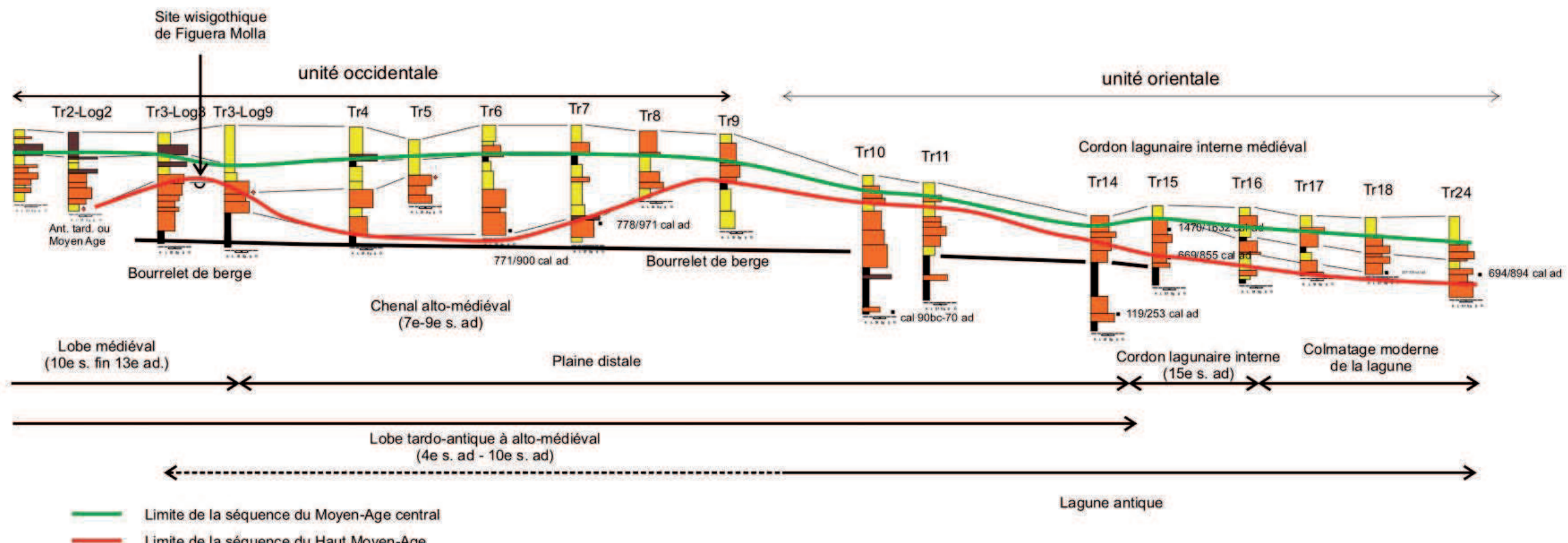

Fig. 4: Séquence alluviale sur le site de Torreilles - La Madraguère.

Fig. 4: Alluvial sequence in Torreilles - La Madraguère excavation. 
tion de faciès de chenaux puis de chenaux et de levées de berge atteste de la mise en place d'un cône distal sableux. Les apports se poursuivent jusqu'au IV ${ }^{\mathrm{e}}$ siècle ap. J.-C., période à laquelle la vitesse de sédimentation moyenne décroît rapidement. Ce changement de dynamique alluviale (passage d'une sédimentation de sables grossiers associée à des environnements de chenal-plaine alluviale proximale) à une sédimentation sablo-limoneuse de plaine distale est le résultat d'une défluviation de l'Agly, documentée par les travaux de la RD83.

\section{2 - AUTOUR DE LA RD 83}

Deux opérations successives ont permis l'observation de l'organisation sédimentaire sur un linéaire total de plus de 4 km (Vignaud, 2008; Passarius et al., 2010). Elles ont permis l'identification de quatre séquences alluviales majeures (fig. 5). La base de la séquence est représentée par des formations lagunaires argilo-sableuses, fréquemment riches en coquilles et en fragments végétaux. Cette séquence est identifiée dans la partie orientale de la zone diagnostiquée, mais s'étend peut-être vers l'ouest où l'épaississement des formations supérieures n'a pas permis son observation. Elle est recouverte par un ensemble de formations sableuses grossières dont l'épaisseur diminue vers l'est. Elles permettent de mettre en évidence l'existence d'un chenal principal peu incisé bordé par deux bourrelets de berge, dont l'un porte un site attribué à la période wisigothique (entre le $\mathrm{v}^{\mathrm{e}}$ et le $\mathrm{VIII}^{\mathrm{e}}$ siècle ap. J.-C.). L'ensemble de cette topographie est colmaté par une formation fluviale à l'ouest et lagunaire à l'est. Celle-ci permet de localiser un cordon interne à «shell hash layer» qui sépare espace fluvial et lagunaire. Cette formation est couronnée par un paléosol faiblement esquissé.

Les données chrono-stratigraphiques obtenues confirment ce découpage. Elles permettent de dater les formations lagunaires entre le $\mathrm{I}^{\mathrm{er}}$ et le $\mathrm{III}^{\mathrm{e}}$ siècle ap. J.-C. La mise en place de la deuxième séquence alluviale est antérieure à la fin du VIII ${ }^{\mathrm{e}}$ siècle ap. J.-C. comme l'indiquent quatre dates radiocarbones obtenues sur des niveaux supérieurs de cette formation. Cette attribution est cohérente avec les données archéologiques et notamment l'identification au niveau de la levée de berge d'une occupation wisigothique ( $\mathrm{V}^{\mathrm{e}}-\mathrm{VIII}{ }^{\mathrm{e}}$ siècle ap. J.-C.). Celle-ci se localise donc au débouché du tracé alto-médiéval de l'Agly dans la lagune de Leucate (fig. 6). La troisième séquence alluviale à dominante sableuse dans la partie ouest peut être attribuée au Moyen Âge central. Elle se raccorde à l'est à un cordon lagunaire attribué à la fin du Xv siècle ap. J.-C.

L'étude complémentaire des sources historiques permet de localiser et de dater les tracés anciens de l'Agly entre la fin du XII ${ }^{\mathrm{e}}$ et la fin du XIII ${ }^{\mathrm{e}}$ siècle ap. J.-C. Elles montrent que sur cet intervalle de temps, l'Agly a adopté un tracé méridien et parcouru un cône alluvial enraciné à l'ouest de Saint-Laurent-de-la-Salanque. En particulier, le secteur qui porte le toponyme d'Agly Vell (fig. 6) et préserve dans le paysage un tracé méandriforme a pu être rattaché à cette étape de fonctionnement. Ce système a cessé de fonctionner à la fin du XIII ${ }^{\mathrm{e}}$ siècle ap. J.-C., probablement entre 1279 et 1280 comme l'atteste la première mention de chenal abandonné à cette dernière date (Puig, 2009).

\section{5 - INTERPRÉTATION}

Sur la base de ces observations directes et d'informations complémentaires issues de la Banque de Données du Sous-Sol et de travaux antérieurs, il est possible de reconstituer la dynamique d'alluvionnement dans la Salanque entre la fin de l'Âge du Bronze et le début de la période Moderne.

La zone nord de la Salanque constitue jusqu'à la fin de l'Âge du Bronze un vaste espace lagunaire qui prolonge vers le sud la lagune de Leucate. L'émersion du secteur de Torreilles, en relation avec les apports de l'Agly est clairement identifiée sur les sites de La Madraguère et de Gorg d'en Guillem. Un premier lobe alluvial commence donc à s'édifier au sud de la Salanque vers $\sim 1000$ av. J.-C. L'occupation attribuée au Bronze final sur le site de La Madraguère s'inscrit bien dans ce schéma et est également cohérente avec la découverte d'un site de l'Âge du Bronze IIIa (1050-900 av. J.-C.) enfoui au lieu-dit Eixugador (Kotarba et al., 2008). Non loin, la présence en sub-surface d'une occupation antique datée entre le ${ }^{\text {I }}{ }^{\text {er }}$ siècle av. J.-C et le $\mathrm{IV}^{\mathrm{e}}$ siècle ap. J.-C. au lieu-dit Les Parrudes s'inscrit également dans ce schéma. L'abandon du site sur lequel des plombs de pêche ont été retrouvés pourrait être une conséquence du déplacement de l'Agly. Vers le $\mathrm{IV}^{\mathrm{e}}$ siècle ap. J.-C., la vitesse de sédimentation se réduit drastiquement sur le site, en relation avec la migration de l'Agly vers le nord. Le cours d'eau débute alors l'édification d'un second lobe au niveau de SaintLaurent-de-la-Salanque. Cette date de mise en place est confirmée par une datation radiocarbone obtenue sur des formations sableuses de prodelta dans la lagune de Leucate par Buscail-Martin (1978) entre la fin du $\mathrm{III}^{\mathrm{e}}$ et le $\mathrm{VII}^{\mathrm{e}}$ siècle ap. J.-C. (recalibrée par nos soins). Ce lobe est fonctionnel durant le Haut Moyen Âge et probablement jusqu'à la fin du IX ${ }^{\mathrm{e}}$ siècle ap. J.-C. L'occupation tardoantique à wisigothique de Figuera Molla $\left(\mathrm{V}^{\mathrm{e}}-\mathrm{VIII}^{\mathrm{e}}\right.$ siècle ap. J.-C.) est installée sur une levée de berge en bordure de l'exutoire de l'Agly dans la lagune de Leucate. L'habitat est recouvert par 1,30 $\mathrm{m}$ de sédiments attribués à la seconde partie du Moyen Âge. À cette date, l'Agly débute l'édification du lobe de Saint-Hippolyte plus au nord. Les sources historiques (Puig, 2009) permettent d'identifier deux phases de construction: une phase précoce à l'est autour du lieu-dit Conangle $\left(\mathrm{X}^{\mathrm{e}}-\mathrm{fin} \mathrm{XII}^{\mathrm{e}}\right.$ siècle ap. J.-C. ?) puis une phase tardive entre le $\mathrm{XII}^{\mathrm{e}}$ siècle et la fin du XIII ${ }^{\mathrm{e}}$ siècle ap. J.-C. L'abandon de ce lobe se fait aux environs de cette période et plus probablement autour de 1280 ap. J.-C. L'Agly abandonne définitivement son tracé septentrional et construit alors son lobe actuel (lobe du Barcarès). La délimitation de l'espace lagunaire finimédiéval (RD83 tranchée 15) suggère que le colmatage de la partie sud de la lagune est tardif et synchrone des premiers effets du Petit Age Glaciaire (fin XIII - début $X{ }^{e}$ siècle ap. J.-C.) et se prolonge durant une partie du 


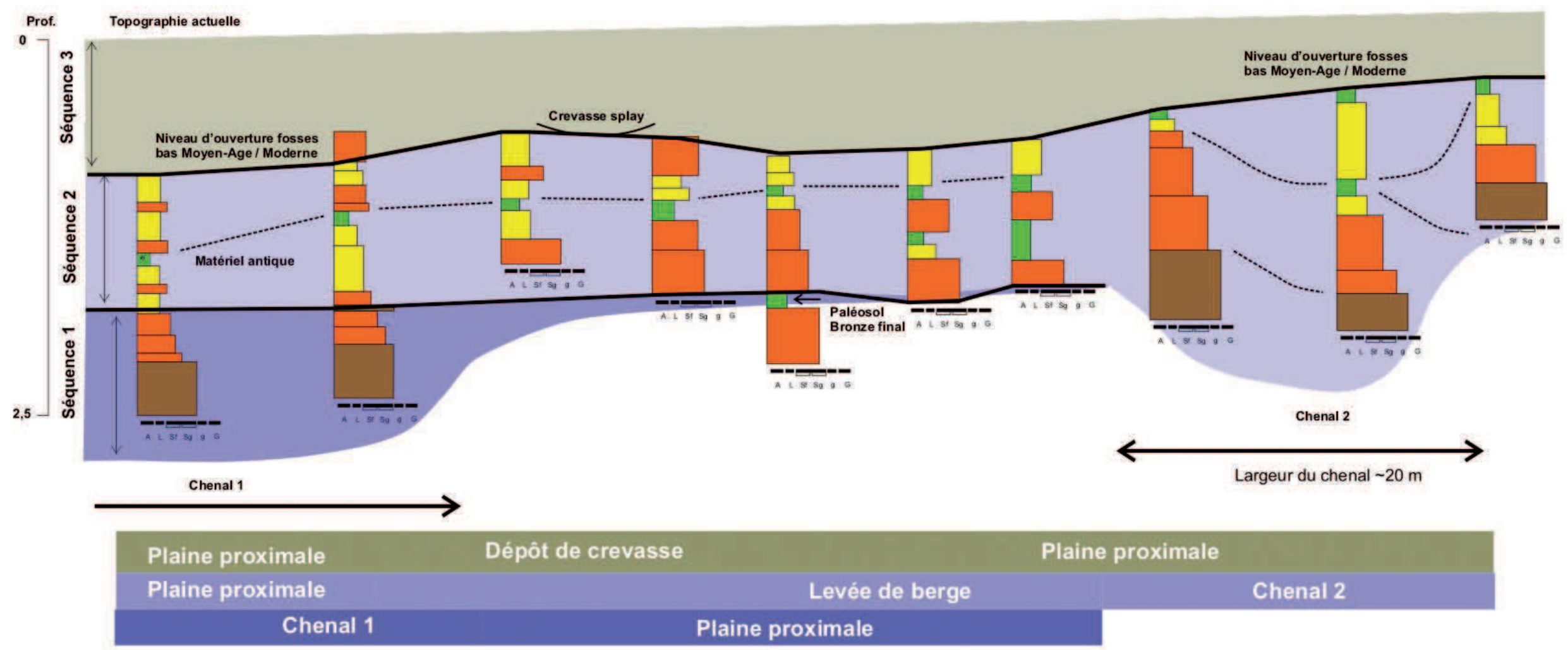

Fig. 5: Séquence alluviale sur le diagnostic de la RD 83.

Fig. 5: Alluvial sequence in the RD 83 test pit. 


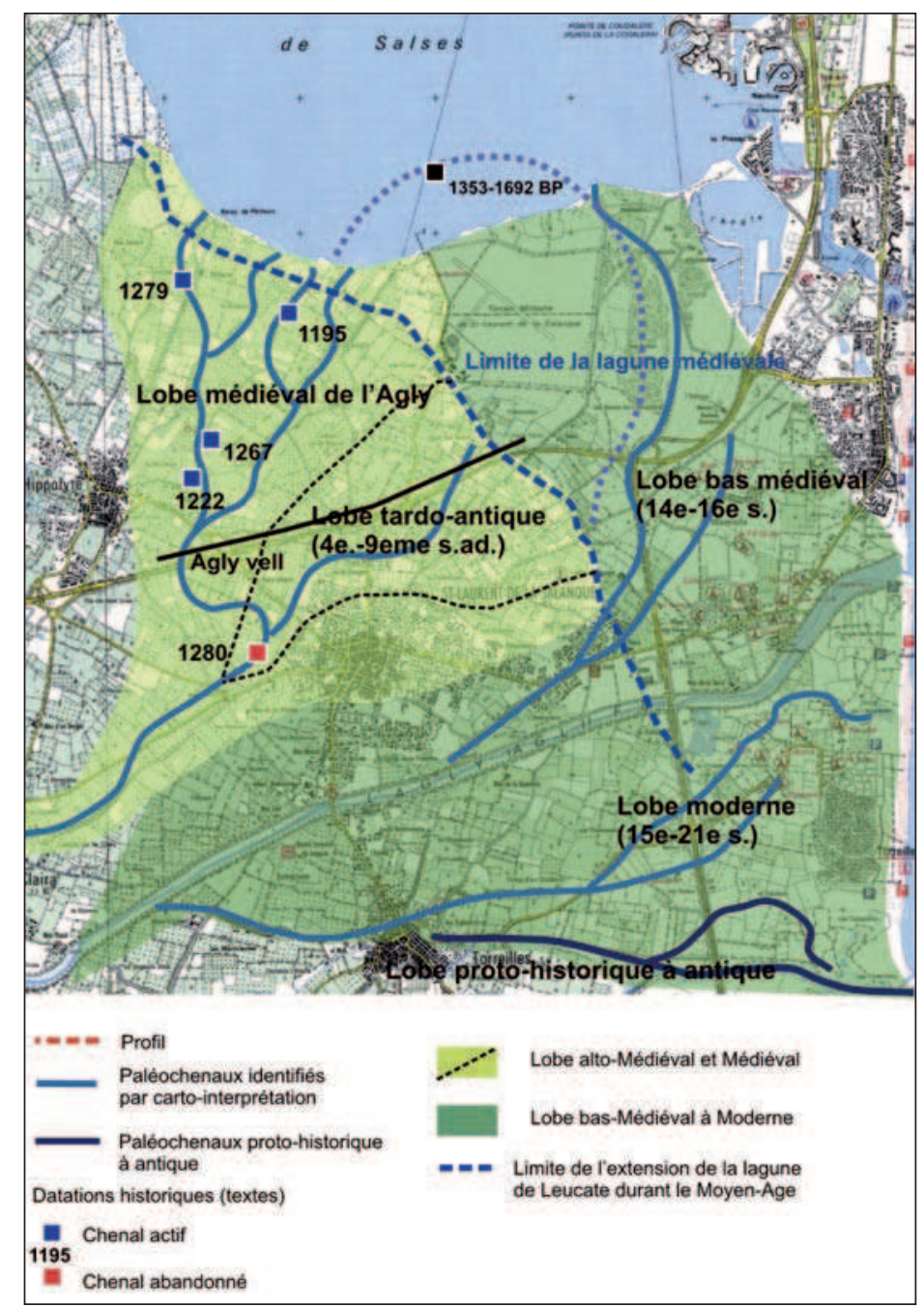

Fig. 6: Carte des lobes alluviaux de l'Agly entre l'an 1000 av. J.-C. et l'actuel.

Fig. 6: Map of Agly River alluvial lobes between 1000 years BC and present.

$\mathrm{XV}^{\mathrm{e}}-\mathrm{XVI}^{\mathrm{e}}$ siècle ap. J.-C. L'endiguement de l'Agly amorcé dans la première moitié du XIV ${ }^{\mathrm{e}}$ siècle ap. J.-C. (Puig, 2009), a probablement favorisé la stabilisation de la zone amont du lobe du Barcarès et le colmatage rapide de la partie sud de la lagune de Leucate.

\section{6 - DISCUSSION}

L'évolution de la plaine de la Salanque apparait, à la lumière des travaux géoarchéologiques récents, comme beaucoup plus complexe que ne le suggéraient les travaux antérieurs. L'utilisation couplée de données géomorphologiques, archéologiques et historiques permet la reconstitution sur les trois derniers millénaires des dynamiques sédimentaires et des transformations des paysages avec une précision parfois séculaire. Loin d'une simple progradation régulière des formations continentales sur le littoral et les espaces lagunaires, l'architecture de la basse plaine montre une organisation complexe qui doit être abordée à deux échelles pour rendre compte de la relation entre processus de construction de la plaine et répartition/préservation de l'information archéologique.
La première, qui a principalement été tentée dans ce travail, est celle de l'organisation des lobes alluviaux à l'échelle de la plaine. Au cours des trois derniers millénaires, le déplacement de l'Agly est marqué par deux périodes. La première correspond à un glissement progressif du sud vers le nord, associée à l'édification successive des lobes de Torreilles, Saint-Laurent-de-laSalanque et de Saint-Hippolyte. Cette tendance avait déjà été observée par Calvet et al. (2002) qui l'ont interprété en terme de contrôle tectonique de la faille de la Têt. Cette interprétation semble contredite par la mise en place du dernier lobe du Barcarès au cours de la deuxième phase d'évolution récente. Il semble que le facteur déterminant de l'architecture sédimentaire à cette échelle soit les processus autocycliques de compensation latérale (lateral shift, Aslan et al., 2005). La chronologie de la mise en place des lobes contrôle à cette échelle le potentiel archéologique.

La deuxième échelle de travail est celle de l'architecture interne du lobe et de son mode d'édification. Les défluviations secondaires, mais aussi les vitesses d'aggradation sont un facteur déterminant de la préservation de l'information archéologique. Ainsi, toujours sur le lobe de Torreilles, les sites proto-historiques sont-ils enfouis sous 
une épaisseur comprise entre 2,40 et $0,80 \mathrm{~m}$ qui correspond à deux épisodes de dépôts différents: dépôts de chenaux-plaine d'inondation proximale protohistorique et antique d'une part, et de plaine distale du bas Moyen Âge d'autre part. Si le second épisode est favorable dans son ensemble à la préservation de l'information archéologique du fait de la faible énergie du milieu, il n'en va pas de même pour les occupations proto-historiques qui semblent occuper, comme cela a déjà été observé dans le delta du Rhône par Arnaud-Fassetta (2000) ou dans le delta du Nil par Welsby et al. (2002) préférentiellement dans les zones de levées de berges. Il est alors difficile de trancher entre préservation différentielle liée à un biais taphonomique de type stratinomique (i.e. lié à l'énergie des milieux de dépôts; Lombard, 1978) et des formes d'adaptation aux contraintes du milieu. Un schéma similaire se dessine pour les occupations du Haut Moyen Âge sur le lobe de Saint-Laurent-de-la-Salanque. Seuls des travaux complémentaires, notamment sur les formations à haute énergie pourront permettre de trancher entre les deux mécanismes.

\section{7 - CONCLUSION}

Dans les basses plaines littorales, sur les deltas et cônes associés à des systèmes géomorphologiques à haute énergie, les modalités de construction sont fortement influencées par les défluviations qui commandent la durée de fonctionnement des lobes élémentaires, leur architecture et leur potentiel de préservation de l'information archéologique.

Dans la Salanque, l'Agly a construit quatre lobes principaux successifs par entre 1000 av. J.-C. et aujourd'hui (lobes de Torreilles, de Saint-Laurent-de-la-Salanque, de Saint-Hippolyte et du Barcarès). Ils colmatent une vaste zone lagunaire qui prolonge l'étang de Leucate vers le sud et explique l'absence d'occupations anciennes reconnues en surface dans ce secteur. Le vide de la carte archéologique s'explique donc pour partie par un effet paléogéographique. Toutefois, la mise en place de ces différents lobes est systématiquement associée à des occupations qui tendent à montrer l'appropriation rapide par les sociétés du passé de ces terres nouvelles conquises par les dynamiques alluviales sur les espaces lagunaires. Ces occupations sont aujourd'hui recouvertes par les formations mises en place à partir de la seconde moitié $\mathrm{du} \mathrm{XIII}^{\mathrm{e}}$ siècle $\mathrm{AD}$ qui dépassent généralement $0,60 \mathrm{~m}$ en moyenne et peuvent atteindre $3 \mathrm{~m}$ d'épaisseur. L'absence de sites de surface est donc également le résultat d'un biais taphonomique d'enfouissement.

\section{RÉFÉRENCES BIBLIOGRAPHIQUES}

ARNAUD-FASSETTA G., 2000 - Quatre mille ans d'histoire hydrologique dans le delta du Rhône. De l'âge du Bronze au siècle du nucléaire. Grafigéo, 11. Pôle de recherche pour l'organisation et la diffusion de l'information géographique, Paris, 229 p.

ASLAN A., AUTIN W.J. \& BLUM M.D., 2005 - Causes of river avulsion: insights from the Late Holocene avulsion history of the Mississippi River, USA. Journal of Sedimentary Research, 75 (4) 650-664.
BENECH C. \& MUSSOT R., 1995 - L'influence des interventions humaines sur l'écoulement des eaux et sur les transports solides. L'exemple des Pyrénées-Orientales (France). Annales de Géographie, 104 (581-582), 105-118.

BERGER J.-F., 2011 - Hydrological and post-depositional impacts on the distribution of Holocene archaeological sites: The case of the Holocene middle Rhône River basin, France. Geomorphology, 129 (3-4), 169-182.

BUSCAIL-MARTIN R., 1978 - Evolution holocène et actuelle des conditions de sédimentation dans le milieu lagunaire de SalsesLeucate. Thèse de Doctorat, Université Toulouse 3 Paul Sabatier, Perpignan, $228 \mathrm{p}$.

CALVET M., 1994 - Morphogenèse d'une montagne méditerranéenne : les Pyrénées orientales. Thèse d'État, Université Paris 1 PanthéonSorbonne, Paris, $1177 \mathrm{p}$.

CALVET M., SERRAT P., LEMARTINEL B. \& MARICHAL R., 2002 - Les cours d'eau des Pyrénées orientales depuis 15000 ans: état des connaissances et perspectives de recherches. In J.-P. Bravard \& M. Magny (dir.), Les fleuves ont une histoire: Paléo-environnement des rivières et des lacs français depuis 15000 ans. Archéologie Aujourd'hui. Errance, Paris, 279-294.

CAROZZA J.-M., 1998 - Évolution des systèmes géomorphologiques en contexte orogénique: l'exemple des bassins d'alimentation du Roussillon (Pyrénées Orientales). Approche morphotectonique. Thèse de Doctorat, Université Toulouse 2 Le Mirail, Toulouse, 381 p.

CAROZZA J.-M., 2005 - «La plaine du Roussillon à l'Holocène: de l'évolution paléogéographique à la modélisation prédictive». Rapport de Projet Collectif de Recherche, Service Régional de l'Archéologie de Languedoc-Roussillon, Montpellier, 84 p.

CAROZZA J.-M., PUIG C., ODIOT T. \& VALETTE P., 2010 - La plaine du Roussillon au cours de l'Holocène: acquis de la recherche et perspectives. In X. Delestre \& H. Marchesi (dir.), Archéologie des rivages méditerranéens: 50 ans de recherche: Actes du colloque d'Arles 28-29-30 octobre 2009. Errance \& Ministère de la Culture et de la Communication, Paris, 39-46.

CAROZZA J.-M., 2011 - L'évolution géomorphologique des basses plaines littorales méditerranéennes: un outil de compréhension des biais taphonomiques en archéologie? Méditerranée, 117, 45-51.

CLAUZON G., BERGER G.-M., ALOISI J.-C., GOT H., MONACO A., MARTIN-BUSCAIL R., GADEL F., AUGRIS C. \& MARCHAL J.-P., 1989 - Carte géologique de la France à 1/50000 ( ${ }^{\circ}$ 1091), Perpignan. Notice explicative. BRGM, Orléans, $40 \mathrm{p}$.

CORBAN D., 2007 - Torreilles - Gorg d'en Guillem. Document Final de Synthèse, Direction Régionale des Affaires Culturelles du Languedoc-Roussillon, Montpellier, $30 \mathrm{p}$.

DUVAIL C., 2004 - Expression des facteurs régionaux et locaux dans l'enregistrement sédimentaire d'une marge passive. Exemple de la marge du Golfe du Lion, étudiée selon un continuum terre-mer. Thèse de Doctorat, Université Montpellier 2, Montpellier, $261 \mathrm{p}$.

KOTARBA J., CALSTELLVI G. \& MAZIÈRE F. 2008. - Les Pyrénées-Orientales. Carte archéologique de la Gaule, 66. Académie des Inscriptions et Belles-Lettres, Ministère de l'Éducation Nationale, Ministère de la Recherche, Ministère de la Culture et de la Communication \& Maison des Sciences de l'Homme, Paris, 712 p.

LOMBARD A., 1978 - Sedimentology. In R.W. Fairbridge \& J. Bourgeois (eds.), Encyclopedia of Sedimentology. Dowden, Hutchinson \& Ross, Stroudsburg, 703-707.

MARICHAL R., REBE I. \& TRETON R., 1997 - La Transformation du milieu géomorphologique de la plaine du Roussillon et ses conséquences sur son occupation: premiers résultats. In J. Burnouf, J.-P. Bravard \& G. Chouquer (eds.), La dynamique des paysages protohistoriques, antiques, médiévaux et modernes. XVII's rencontres internationales d'archéologie et d'histoire d'Antibes: actes des rencontres, 19-20-21 octobre 1996. Éditions APDCA, Sophia-Antipolis, 272-283.

PASSARRIUS O., CAROZZA J.-M., KOTARBA J., ILLES P. \& PORRA-KETUNI., 2010 - Élargissement de la RD83. Communes de Saint-Laurent-de-la-Salanque et du Barcarès. Document Final de Synthèse, Direction Régionale des Affaires Culturelles du Languedoc-Roussillon, Montpellier, $36 \mathrm{p}$.

PUIG C., 2009 - Les prémices du Petit Age Glaciaire en Roussillon à travers le prisme des sources écrites. Archéologie du Midi Médiéval, 27, 191-205.

PUIG C. \& CAROZZA J.-M., 2012 - Les changements de tracés des cours d'eau d'après les sources historiques et géomorphologiques dans la plaine du Roussillon depuis le XII siècle: approche théorique et premiers résultats. In V. Ropiot, C. Puig \& F. Mazière (eds.), Les plaines littorales en Méditerranée nord-occidentale. Éditions Monique Mergoil, Montagnac, 297-311.

PY M., 1993 - Les Gaulois du Midi: De la fin de l'âge du Bronze à la Conquete romaine. La mémoire du temps. Hachette, Paris, 288 p. 
SERRAT P., 2000 - Genèse et dynamique d'un système fluvial méditerranéen: le bassin de l'Agly (France). Thèse de Doctorat, Université de Perpignan, Perpignan, 790 p.

TESSON M., LABAUNE C., GENSOUS B \& DELHAYE-PRAT V., 2010 - Quaternary compound incised valleys of the Roussillon coast (SE France): correlation of seismic data with core data. Bulletin de la Société géologique de France, 181 (2), 183-196.

VIAL J., 2009 - Torreilles - La Madraguère. Document Final de Synthèse, Direction Régionale des Affaires Culturelles du LanguedocRoussillon, Montpellier, $57 \mathrm{p}$
VIGNAUD A., 2008 - RD 83 - Phase 1. Service Régional de l'Archéologie de Languedoc-Roussillon, Montpellier, 42 p.

WELSBY D.A., MACKLIN M.G. \& WOODWARD J.C., 2002

- Human responses to Holocene environmental changes in the Northern Dongola Reach of the Nile, Sudan. In R.F. Friedman (ed.) Egypt and Nubia: Gifts of the Desert. The British Museum Press, London, 28-38. 